\title{
Air-core fiber distribution of hybrid vector vortex-polarization entangled states
}

\author{
Daniele Cozzolino, ${ }^{\mathrm{a}}$ Emanuele Polino, ${ }^{\mathrm{b}}$ Mauro Valeri, ${ }^{\mathrm{b}}$ Gonzalo Carvacho, ${ }^{\mathrm{b}}$ Davide Bacco, ${ }^{\mathrm{a}}$ \\ Nicolò Spagnolo, ${ }^{b}$ Leif K. Oxenløwe, ${ }^{a}$ and Fabio Sciarrino ${ }^{\mathrm{b}, \mathrm{c}, \boldsymbol{}}$ \\ ${ }^{a}$ Technical University of Denmark, CoE SPOC, Department of Photonics Engineering, Lyngby, Denmark \\ 'Sapienza Università di Roma, Dipartimento di Fisica, Roma, Italy \\ ${ }^{c}$ Consiglio Nazionale delle Ricerche, Istituto dei Sistemi Complessi, Roma, Italy
}

\begin{abstract}
Entanglement distribution between distant parties is one of the most important and challenging tasks in quantum communication. Distribution of photonic entangled states using optical fiber links is a fundamental building block toward quantum networks. Among the different degrees of freedom, orbital angular momentum (OAM) is one of the most promising due to its natural capability to encode high dimensional quantum states. We experimentally demonstrate fiber distribution of hybrid polarization-vector vortex entangled photon pairs. To this end, we exploit a recently developed air-core fiber that supports OAM modes. High fidelity distribution of the entangled states is demonstrated by performing quantum state tomography in the polarization-OAM Hilbert space after fiber propagation and by violations of Bell inequalities and multipartite entanglement tests. The results open new scenarios for quantum applications where correlated complex states can be transmitted by exploiting the vectorial nature of light.
\end{abstract}

Keywords: orbital angular momentum; quantum communication; structured light; multimode fiber; multipartite entanglement.

Received May 10, 2019; accepted for publication Aug. 5, 2019; published online Aug. 27, 2019.

(C) The Authors. Published by SPIE and CLP under a Creative Commons Attribution 4.0 Unported License. Distribution or reproduction of this work in whole or in part requires full attribution of the original publication, including its DOI.

[DOI: 10.1117/1.AP.1.4.046005]

\section{Introduction}

Quantum communication requires the reliable transmission of quantized information carriers (qubits) among several and spatially separated parties, ${ }^{1}$ toward development of quantum networks. In particular, protocols based on genuine quantum schemes, such as entanglement swapping, ${ }^{2-5}$ superdense coding, ${ }^{6-9}$ and quantum teleportation, ${ }^{10-16}$ have to be adopted in future networks to access communication advantages that would be unattainable by using any classical resource. The key element of these schemes is entanglement, which is one of the most distinctive quantum phenomena, predicted by Einstein, Podolsky, and Rosen ${ }^{17}$ that defy the classical notion of local causality. ${ }^{18}$ Quantum correlations are an essential ingredient for quantum foundations studies and for different quantum information processes. ${ }^{19-22}$ Great interest has been devoted to the coherent distribution through optical fibers of such quantum correlations since it constitutes the cornerstone for the future quantum networks. ${ }^{23-26}$ Several photon degrees of freedom can be

*Address all correspondence to Fabio Sciarrino, E-mail: fabio.sciarrino@uniroma1.it employed for this task, such as frequency, orbital angular momentum (OAM), time, and polarization. ${ }^{25-29}$ In particular, OAM of light is one of the most promising, albeit challenging to manipulate. Photons owning a nonzero OAM are characterized by the azimuthal phase dependence $e^{i \ell \phi}$, where $\ell \hbar$ is the amount of OAM carried by each photon, and $\ell$ is an unbounded integer value representing discrete quantum states. ${ }^{30,31}$ Due to its unbounded nature, OAM has been largely investigated both for classical and quantum communications, being capable of encoding high dimensional quantum states (qudits), which enhance the photon information capacity. ${ }^{32-43}$ Experimental investigations on OAM supporting fibers for classical communications have been reported. ${ }^{32,33,44-46}$ Still, distribution of quantum states through multimode fibers supporting OAM modes is a newborn research field, where only a few experiments have been realized to date. ${ }^{47,48}$ In Ref. 47, high-dimensional quantum states, encoded in weak coherent pulses, have been transmitted and detected through a 1.2-km length air-core fiber at telecom wavelength, demonstrating the feasibility of high-dimensional quantum communication and quantum key distribution protocols. In Ref. 48, a solid core vortex fiber has been exploited, 
demonstrating the possibility of two-dimensional quantum communication with structured photons. Nonetheless, the transmission of entangled photon pairs still needs to be explored.

In this work, we demonstrate distribution of hybrid entanglement between a linearly polarized photon and a vector vortex (VV) beam, i.e., a doughnut-shaped beam with an inhomogeneous polarization pattern, at telecom wavelength. The VV beam is transmitted through a 5-m long air-core fiber, ${ }^{49}$ whose very low mode mixing preserves OAM states and, in turn, hybrid entanglement. This peculiar feature opens up new scenarios and opportunities in quantum communications toward fiberbased quantum networks, enabling the capability to employ high-dimensional quantum states, embedded in the photon polarization and OAM degrees of freedom.

\section{Vector Vortex Beam and Hybrid Entanglement Generation}

Vector vortex beams constitute a special class of vector beams, which are characterized by an inhomogeneous polarization distribution over their transverse profile..$^{50}$ In particular, a VV beam has an azimuthally varying polarization pattern, surrounding a central optical singularity. ${ }^{51-53}$ Due to their distinctive polarization distributions, VV beams have shown unique features, making them appealing for different research purposes, e.g., microscopy, ${ }^{54}$ optical trapping, ${ }^{55,56}$ metrology, ${ }^{57,58}$ nanophotonics, ${ }^{59}$ and communication. ${ }^{60-67}$ Formally, the state $|R, \ell\rangle$ $(|L, \ell\rangle)$ describes a photon with uniform right (left) circular polarization carrying $\ell \hbar$ of OAM, and a VV beam can be conveniently described by a nonseparable superposition of polarization-OAM eigenmodes. In this superposition, OAM quanta carried by the photons define the order $m$ of the VV beam. In particular, a VV beam belongs to a Hilbert space spanned by states $\{|R, m\rangle,|L,-m\rangle\} .{ }^{53}$ For instance, if $m=1$ and if we consider equally distributed superpositions $\left|r_{1}\right\rangle=(|R,+1\rangle+$ $|L,-1\rangle) / \sqrt{2}$ and $\left|a_{1}\right\rangle=(|R,+1\rangle-|L,-1\rangle) / \sqrt{2}$, radially and azimuthally polarized beams are obtained..$^{1,52}$

Here, we experimentally demonstrate fiber distribution of a VV-polarization entangled photon state. The conceptual scheme of the experiment is reported in Fig. 1. A polarization-VV beam entangled photon pair is generated from an initial polarization entangled pair (blue ribbon). Only one photon of the pair encodes the VV state (green ribbon). Subsequently, the VV beam is coupled and transmitted through the 5-m long air-core fiber and then measured together with the linearly polarized photon. In our case, VV beams of order $m=7$ are generated, whose expressions are

$\left|r_{7}\right\rangle=\frac{|R,+7\rangle+|L,-7\rangle}{\sqrt{2}}$,

$\left|a_{7}\right\rangle=\frac{|R,+7\rangle-|L,-7\rangle}{\sqrt{2}}$.

The polarization patterns associated to states $\left|r_{7}\right\rangle$ and $\left|a_{7}\right\rangle$ are shown in Fig. 1(a). In the following, we will refer to $\left|r_{7}\right\rangle$ and $\left|a_{7}\right\rangle$ as $|r\rangle$ and $|a\rangle$, respectively. The experimental apparatus is reported in Fig. 2. Polarization entangled photon pairs, $\left(|H\rangle|V\rangle+\mathrm{e}^{i \phi}|V\rangle|H\rangle\right) / \sqrt{2}$, are generated at $1550 \mathrm{~nm}$ wavelength by a periodically poled potassium titanyl phosphate (ppKTP) crystal placed into a polarization Sagnac interferometer and pumped with a continuous-wave laser at $775 \mathrm{~nm}$. Indeed, for fiber-based quantum communication, it is important to exploit photons within the C-band (1530 to $1565 \mathrm{~nm}$ ), where optical fibers show minimal losses. The relative phase $\phi$ of the entangled state is controlled to generate the singlet state

$|\psi\rangle_{s}=\frac{1}{\sqrt{2}}\left(|H\rangle_{1}|V\rangle_{2}-|V\rangle_{1}|H\rangle_{2}\right)$,

where the subscripts 1 and 2 indicate the two interferometer output modes. According to the notation in Fig. 2, photons along output mode 2 impinge on a vortex plate (VP), adding OAM order $m=7$. The VP can be considered as a nontunable $q$-plate $(\mathrm{QP})$, i.e., a device that couples the polarization and the OAM of a single photon ${ }^{68}$ Specifically, in the circular polarization basis $\{|R\rangle,|L\rangle\}$, the action of a $\mathrm{QP}$ with topological charge $q$, on a single photon of OAM order $k$, is described by the
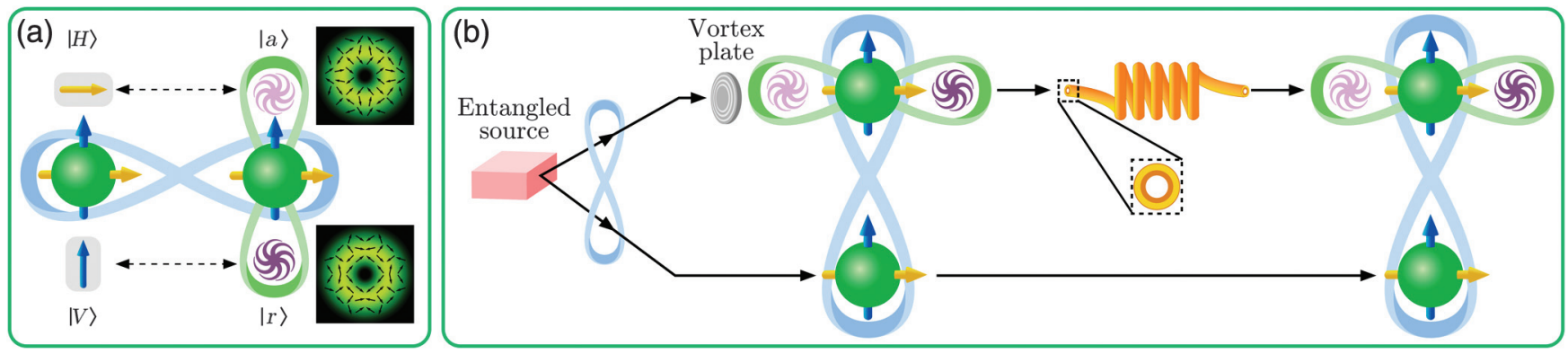

Fig. 1 Hybrid entangled state transmission. (a) Hybrid VV-polarization entangled photon pair generated in the experiment: entanglement in polarization of the photon pair (blue ribbon) and entanglement between polarization and OAM of the single photon (green ribbon, VV state) are sketched. The inhomogeneous polarization patterns of the VV state $|r\rangle$ (bottom) and $|a\rangle$ (up) are explicitly shown. (b) Schematic of the experiment: hybrid VV-polarization entangled state is generated by an initial polarization entangled photon pair. One photon of the pair encodes the VV state by the action of a VP. The VV beam is transmitted through the air-core fiber. Finally, state detection shows that hybrid VV-polarization entanglement (blue and green ribbons) is preserved after fiber transmission. 


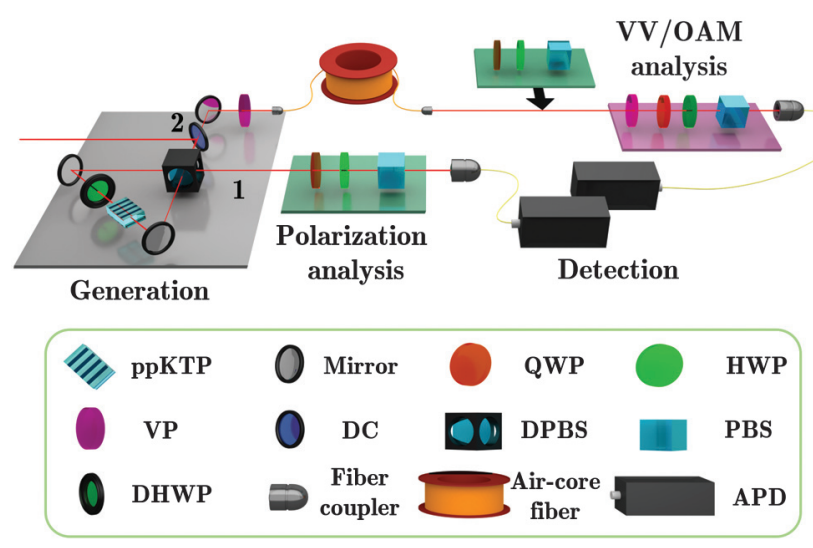

Fig. 2 Experimental apparatus for the generation, distribution and analysis of the hybrid entangled states. Pairs of telecom polarization entangled photons are generated by exploiting a periodically poled titanyl phosphate crystal (ppKTP) in a Sagnac interferometer, which contains a dual-wavelength polarizing beam splitter (DPBS) and a dual half-wave plate (DHWP). Photons exiting along mode 1 are sent to a polarization analysis stage, composed of a QWP, an HWP and a PBS. Photons along mode 2 pass through a dichroic mirror (DC), which separates the pump from the photons. Photons in mode 2 impinge on a VP to generate a VV beam state and, in turn, the desired hybrid entangled state. The VV states are coupled to an air-core fiber and then measured with an OAM-polarization analysis stage composed of a second VP followed by a polarization analysis setup. To perform the measurements on the polarization and OAM degrees of freedom independently, an additional polarization measurement stage has to be inserted before the OAM-toGaussian conversion regulated by the second VP. Finally, both photons are coupled into single-mode fibers linked to avalanche photodiode single photon detectors (APDs).

transformation: $|L, k\rangle \stackrel{\mathrm{QP}}{\rightarrow}|R, k+2 q\rangle$ and $|R, k\rangle \stackrel{\mathrm{QP}}{\rightarrow}|L, k-2 q\rangle$. In our case, $m=2 q=7$ and the initial OAM order is $k=0$. Exploiting the spin-orbit coupling, we generate the VV state $|r\rangle$ or $|a\rangle$ depending on the input polarization state, $|H\rangle$ or $|V\rangle$, respectively. ${ }^{69,70}$ Thus, the polarization entangled singlet state is transformed into the hybrid entangled state

$|\psi\rangle=\frac{1}{\sqrt{2}}\left(|H\rangle_{1}|a\rangle_{2}-|V\rangle_{1}|r\rangle_{2}\right)$.

The versatility of our experimental approach is based on full control of each degree of freedom through suitable optical components, allowing the preparation of the desired hybrid VV-polarization entangled state [Eq. (4)].

\section{Hybrid Entanglement Distribution and Measurement}

The main purpose of our work is to prove the feasibility of entanglement distribution with OAM states transmitted through an air-core fiber. In particular, we demonstrate that the coherence of the complex hybrid entangled state in Eq. (4) is preserved. This is possible since the states $|r\rangle$ and $|a\rangle$ are superposition of antialigned states, i.e., spin (polarization) and OAM with opposite sign, hence the VV beams are degenerate in time along the fiber transmission. ${ }^{49}$ Indeed, an eventual nondegeneracy of those states could impair the coherence of the VV states and, therefore, of the entangled pair. Different theoretical analysis and measurements can be performed to assess the fidelity of the transmitted states and to quantify entanglement, depending on the requirements in the certification process. ${ }^{71}$ In this work, the quality of the transmitted states is measured through the standard tomography processes ${ }^{72}$ and their entanglement is certified through $\mathrm{CHSH}$-like inequality violations ${ }^{73}$ and tripartite entanglement tests. ${ }^{74-79}$

\subsection{Source State}

As a first step, we characterize the initial polarization entangled state in Eq. (3). To fully determine the quality of the state generated by the ppKTP source, we perform a quantum state tomography within the polarization space of the two photons. The measurements are implemented by collecting twofold detection after two polarization analysis stages placed along each output mode of the source. The obtained tomography is shown in Fig. 3(a), where the relative fidelity with respect to the ideal singlet state is $F_{s}=(93.5 \pm 0.2) \%$. Furthermore, we carry out a nonlocality test obtaining as the maximum value $S$ of the CHSH inequality $S_{s}^{(\text {raw })}=2.67 \pm 0.01 .^{73}$ Subtracting the accidental coincidences from $S_{s}^{\text {(raw) }}$, such parameter becomes $S_{s}=2.68 \pm 0.01$.

\subsection{Hybrid Entangled State (HyEnt)}

Subsequently, we consider the global hybrid VV-polarization entangled state in Eq. (4) and measure the twofold detection after the VV state propagation through the air-core fiber. The fiber structure allows the transmission of OAM modes with very low mode mixing among them. It is composed by a central air core surrounded by a high refractive index ring, creating a large refractive index step that shapes the field of the modes, allowing for their guidance. The fiber we used supports OAM modes with $\ell= \pm 5, \pm 6, \pm 7$ and has shown to achieve $1 \mathrm{~dB} / \mathrm{km}$ losses. Further details and specifications on the fiber are reported in Refs. 47 and 49. In our experiment, we have decided to work with modes $\ell= \pm 7$, achieving a coupling efficiency $\eta=0.5$. At this stage, we consider that the entangled two qubit state lies in a four-dimensional space spanned by the basis $\left\{|H\rangle_{1}|a\rangle_{2},|V\rangle_{1}|a\rangle_{2},|H\rangle_{1}|r\rangle_{2},|V\rangle_{1}|r\rangle_{2}\right\}$, which is composed by the polarization of photon 1 and the VV states of photon 2 . The qubit encoded in photon 1 is measured by a polarization analysis stage (green platform in Fig. 2). Conversely, the measurements of the VV qubit, i.e., photon 2, are implemented by a VP, identical to the one used in the generation process, and a polarization analysis stage (purple platform in Fig. 2). The VP converts back the VV beam to the fundamental Gaussian-like mode, restoring the initial polarization state for photon 2 before impinging on the first VP. In this way, the VV states $|r\rangle$ and $|a\rangle$ are directly mapped into polarization states $|H\rangle$ and $|V\rangle$, which are measured with the usual set of quarter-wave plate (QWP), halfwave plate (HWP), and a polarization beam splitter (PBS) (see Fig. 2). ${ }^{61,69,70}$ State tomography in the four-dimensional space is reported in Fig. 3(b). We consider as a target state the ideal evolution of the density matrix describing the experimental state generated by the source. The resulting fidelity between such state and the state measured after the fiber propagation is $F_{h}=$ $(97.9 \pm 0.2) \%$. Furthermore, we observe violation of the $\mathrm{CHSH}$ inequality, obtaining the value $S_{h}^{\text {(raw) }}=2.62 \pm 0.03$ for raw data 
(a) Source entanglement
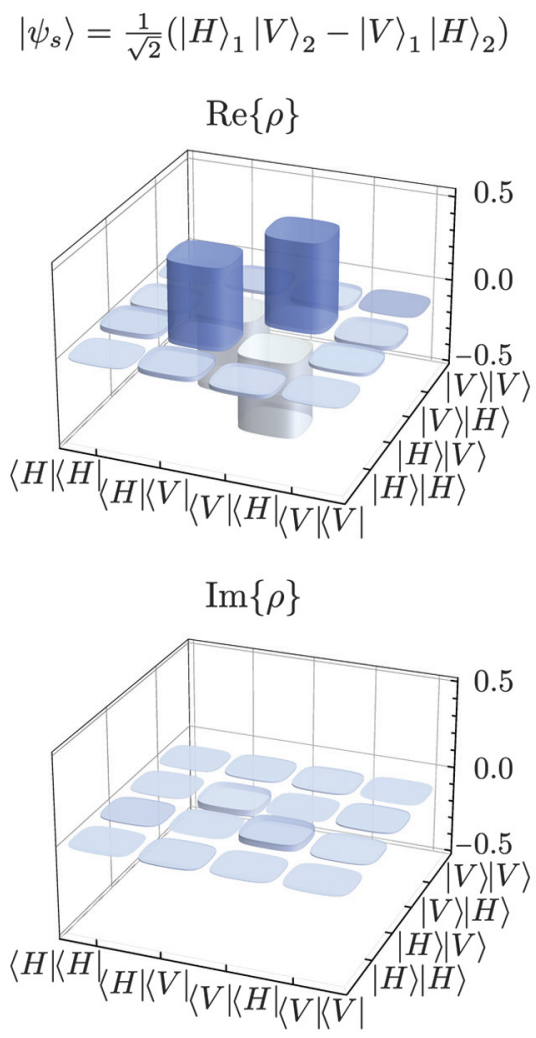

(b) Hybrid entanglement

$$
|\psi\rangle=\frac{1}{\sqrt{2}}\left(|H\rangle_{1}|a\rangle_{2}-|V\rangle_{1}|r\rangle_{2}\right)
$$
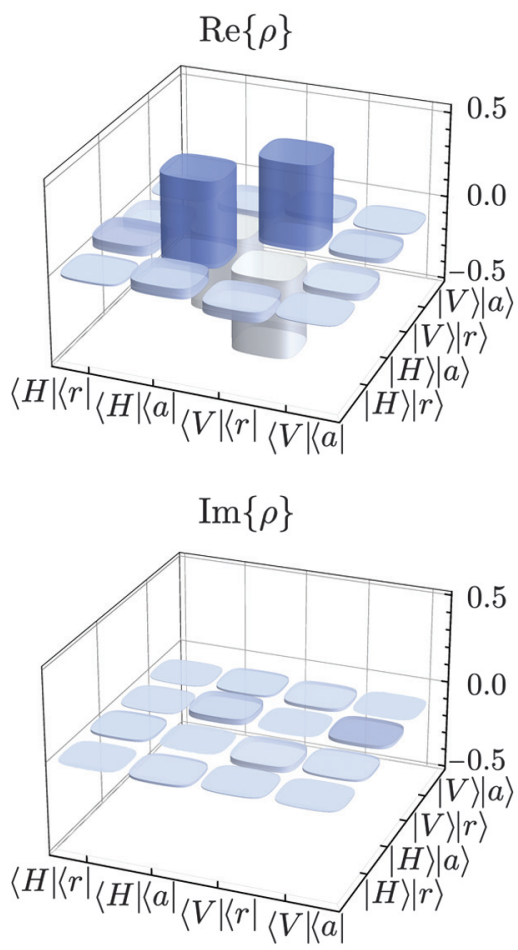

(c) Intra-system entanglement
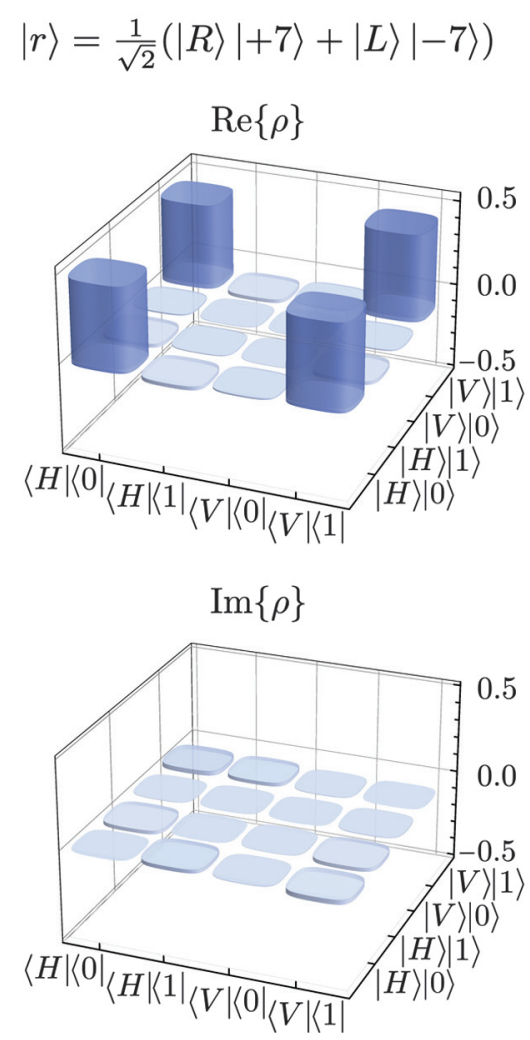

Fig. 3 Two-qubit quantum tomographies. (a) Real (top) and imaginary (bottom) parts of the measured density matrix of the polarization entangled state generated by the source, before conversion in OAM. (b) Real (top) and imaginary (bottom) parts of the measured density matrix of the two-photon VV-polarization entangled state after the transmission of photon 2 through the OAM fiber. (c) Real (top) and imaginary (bottom) parts of the measured density matrix of the VV state on photon 2, transmitted through the OAM fiber. The OAM states $|0\rangle$ and $|1\rangle$ in the tomography are defined by the relations: $|0\rangle \equiv(|+7\rangle+|-7\rangle) / \sqrt{2}$ and $|1\rangle \equiv i(|-7\rangle-|+7\rangle) / \sqrt{2}$. Real and imaginary parts of the experimental density matrices are reconstructed via quantum state tomographies.

and value $S_{h}=2.67 \pm 0.03$ by subtracting for accidental coincidences, thus violating by 21 and 22 standard deviations the separable limit $S=2$, respectively.

\subsection{Intrasystem Entangled State (Intra)}

Now, we focus on the VV state embedded in photon 2 and its transmission through the air-core fiber. Such analysis quantifies the quality of the VV beam state generation, transmission through the air-core fiber, and conversion to the fundamental Gaussian mode. The single photon VV states $|r\rangle$ and $|a\rangle$, Eqs. (1) and (2), are maximally entangled in the OAM and polarization degrees of freedom. They correspond to singleparticle entanglement states, referred to as intrasystem entanglement. The nonseparability between polarization and OAM states is not related to nonlocal properties since they are relative to the same physical system. However, Bell-like inequalities can be exploited to demonstrate the single-particle entanglement, ruling out models that assume realism and noncontexuality of commuting observables, relative to such systems ${ }^{80-83}$ Hence, we certify the presence of intrasystem entanglement carrying out quantum state tomography and performing CHSH-like inequality in the space of polarization and OAM degrees of freedom of photon 2. Horizontally polarized heralded single photons are sent to the VP to conditionally prepare state $|r\rangle$ for photon 2. The measurements on the polarization and the OAM degrees of freedom of photon 2 are performed independently. For this purpose, two cascaded measurement stages are needed (green and purple platforms in Fig. 2). A first stage (HWP, QWP, and PBS) performs the polarization analysis (green platform in Fig. 2). The second stage composed of a VP and a polarization analysis (purple platform in Fig. 2) measures the photon state in the OAM space. As before, the VP maps the information encoded in OAM to a polarization state, which is then measured. Finally, before detection the photon is coupled to a single-mode fiber, tracing out all OAM contributions different from the zero order. The measured quantum state tomography is shown in Fig. 3(c), and the relative fidelity calculated with respect to the Bell state $\left|\Phi^{+}\right\rangle$ is $F_{i}=(99.4 \pm 0.6) \%$. The corresponding parameters $S_{i}$ obtained from the CHSH-like inequality violations are $S_{i}^{\text {(raw) }}=$ $2.76 \pm 0.05$ and $S_{i}=2.82 \pm 0.05$. The set of CHSH violations 
Table $1 \mathrm{CHSH}$ violations. The $\mathrm{CHSH}$ violation parameters obtained from raw data $\left(S^{\text {raw }}\right)$ and by subtracting for accidental coincidences $(S)$ are reported for the polarization entangled state generated by the source, the hybrid VV-polarization entangled state (HyEnt), and the intrasystem entangled VV state embedded in the photon 2 and transmitted through the air-core fiber (intra).

\begin{tabular}{lccc} 
State & Measurement time & $S^{(\text {raw })}$ & $S$ \\
\hline Source & $160 \mathrm{~s}$ & $2.67 \pm 0.01$ & $2.68 \pm 0.01$ \\
HyEnt & $2560 \mathrm{~s}$ & $2.62 \pm 0.03$ & $2.67 \pm 0.03$ \\
Intra & $1920 \mathrm{~s}$ & $2.76 \pm 0.05$ & $2.82 \pm 0.05$ \\
\hline
\end{tabular}

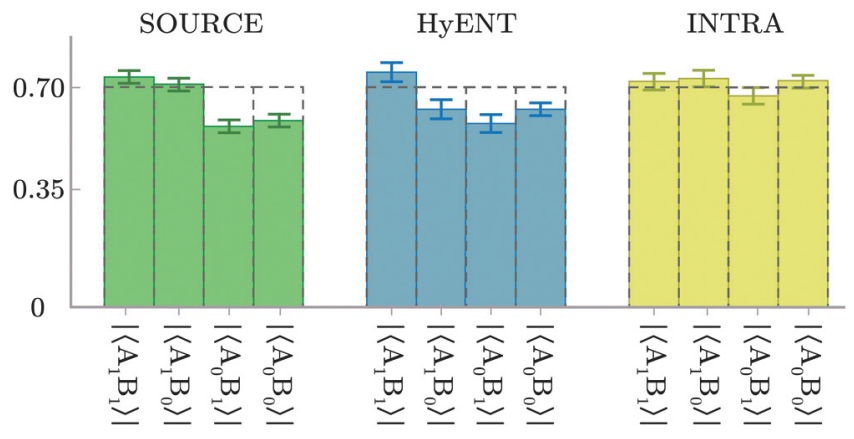

Fig. $4 \mathrm{CHSH}$ measurement operators. Expectation values moduli of the measured operators that maximize the violation of the $\mathrm{CHSH}$ parameter $S=\left\langle A_{1} B_{1}\right\rangle-\left\langle A_{1} B_{0}\right\rangle+\left\langle A_{0} B_{1}\right\rangle+\left\langle A_{0} B_{0}\right\rangle$. The values are relative to the polarization entangled state generated by the source (green bars), the hybrid VV-polarization entangled state (blue bars), and the intrasystem entangled VV state embedded in the photon 2 and transmitted through the air-core fiber (yellow bars). All error bars are due to Poissonian statistics of the measured events.

measured for each state (source, HyEnt, and intra) is summarized in Table 1, and the mean values of the measured operators are shown in Fig. 4.

These results have been carried out for degenerate antialigned states, corresponding to Eqs. (1) and (2), that experience negligible temporal dispersion during propagation. Other classes of OAM modes, enabling to reach higher dimensional systems, can be transmitted through the air-core fiber by adding an appropriate precompensation stage. ${ }^{47}$ Such stage allows one to counteract the modal dispersion due to different effective refractive indices in the fiber. ${ }^{49}$

\subsection{Three Qubits HyEnt}

The previous measurements have independently certified the high fidelity of both the hybrid VV-polarization entangled state and the single photon VV beam state after propagation in the aircore fiber.

We now characterize the hybrid VV-polarization entangled state in Eq. (4) with a different apparatus that does not assume a two-dimensional Hilbert space for photon 2 spanned by $\{|r\rangle,|a\rangle\}$. The measurements on the final state are performed by a polarization analysis stage for photon 1 and by polarization and OAM analysis stages for photon 2, as the one adopted for the intrasystem entanglement characterization (Fig. 2). This apparatus allows one to independently measure the polarization and the OAM component of photon 2 so that the total encoding three-qubit space can be now spanned by the polarization and OAM degrees of freedom in addition to the polarization of photon 1 . Thus, by performing the three-qubit quantum state tomography of the transmitted state (Fig. 5), a final fidelity $F=(88.1 \pm 0.2) \%$ with respect to the ideal state in Eq. (4) is obtained. This shows that the fiber preserves the injected state without adding noise contributions. These results provide additional evidence that the fiber is suitable for the transmission of higher-dimensional quantum states. As for the other cases, also for the three-qubit case we perform a device independent test of the quantum correlations, showing their preservation after fiber transmission of the VV state. First, we test the MerminArdehali-Belinski-Klyshko inequality, ${ }^{74-76}$ which provides an upper bound for contextual hidden variable theories, describing the correlations between observables relative to three qubits:

$\mathcal{M} \equiv\left|\left\langle A_{1} B_{2} C_{2}\right\rangle+\left\langle A_{2} B_{1} C_{2}\right\rangle+\left\langle A_{2} B_{2} C_{1}\right\rangle-\left\langle A_{1} B_{1} C_{1}\right\rangle\right| \leq 2$.

The observables $A_{i}, B_{i}$, and $C_{i}(i=1,2)$ are dichotomic (with eigenvalues \pm 1 ) and relative to the first, the second, and the third qubit, respectively. Violation of such inequality certifies the nonclassical correlations of tripartite states. Furthermore, if a value $\mathcal{M} \geq 2 \sqrt{2}$ is found, models in which quantum correlations are allowed between just two of the three qubits (biseparable quantum models) are ruled out as well. ${ }^{84,85}$ The state Eq. (4) in the three-qubit space is able to reach the algebraic value of $\mathcal{M}=4$ by choosing the operators: $A_{1}=-\sigma_{z}^{A}$, $A_{2}=\sigma_{x}^{A}, B_{1}=-\sigma_{z}^{B}, B_{2}=\sigma_{x}^{B}$, where $\sigma_{i}(i=x, z)$ are the Pauli operators relative to photons $1(A)$ and $2(B)$ in the polarization in basis $\{|H\rangle,|V\rangle\}$; and $C_{1}=\sigma_{z}^{C}, C_{2}=\sigma_{x}^{C}$, where the Pauli operators are in the OAM basis $\{|0\rangle \equiv(|+7\rangle+$ $|-7\rangle) / \sqrt{2},|1\rangle \equiv i(|-7\rangle-|+7\rangle) / \sqrt{2}\}$ relative to photon 2 . Measuring such operators after the VV state transmission and calculating the parameter $\mathcal{M}$, we obtain $\mathcal{M}^{\text {(raw) }}=3.43 \pm$ 0.04 from raw data, and the value $\mathcal{M}=3.53 \pm 0.04$ by subtracting accidental coincidences. In this way, we violated the classical bound by 35 and 38 standard deviations and the quantum biseparable bound by 15 and 17 standard deviations, respectively.

Finally, we further study the correlation of the state in Eq. (4) by performing a Hardy test, ${ }^{77,78}$ recently generalized in a suitable form for more than two parties by Ref. 79. Given a system with certain null correlation probabilities, a paradox arises when other events are automatically forbidden in the framework of noncontextual hidden variable models while they can happen within a quantum context. Since experimentally measuring null probabilities represents a difficult task, Hardy logical contradictions can be conveniently mapped into more general inequalities. In Ref. 79, an extended multiparty version of Hardy's paradox is proposed, leading to an inequality that for three qubits reads

$$
\begin{aligned}
\mathcal{H} \equiv & P\left(A_{1} A_{2} A_{3}\right)-P\left(A_{1} B_{2} B_{3}\right)-P\left(A_{1} \bar{B}_{2} \bar{B}_{3}\right) \\
& -P\left(B_{1} A_{2} B_{3}\right)-P\left(\bar{B}_{1} A_{2} \bar{B}_{3}\right)-P\left(B_{1} B_{2} A_{3}\right) \\
& -P\left(\bar{B}_{1} \bar{B}_{2} A_{3}\right) \leq 0,
\end{aligned}
$$




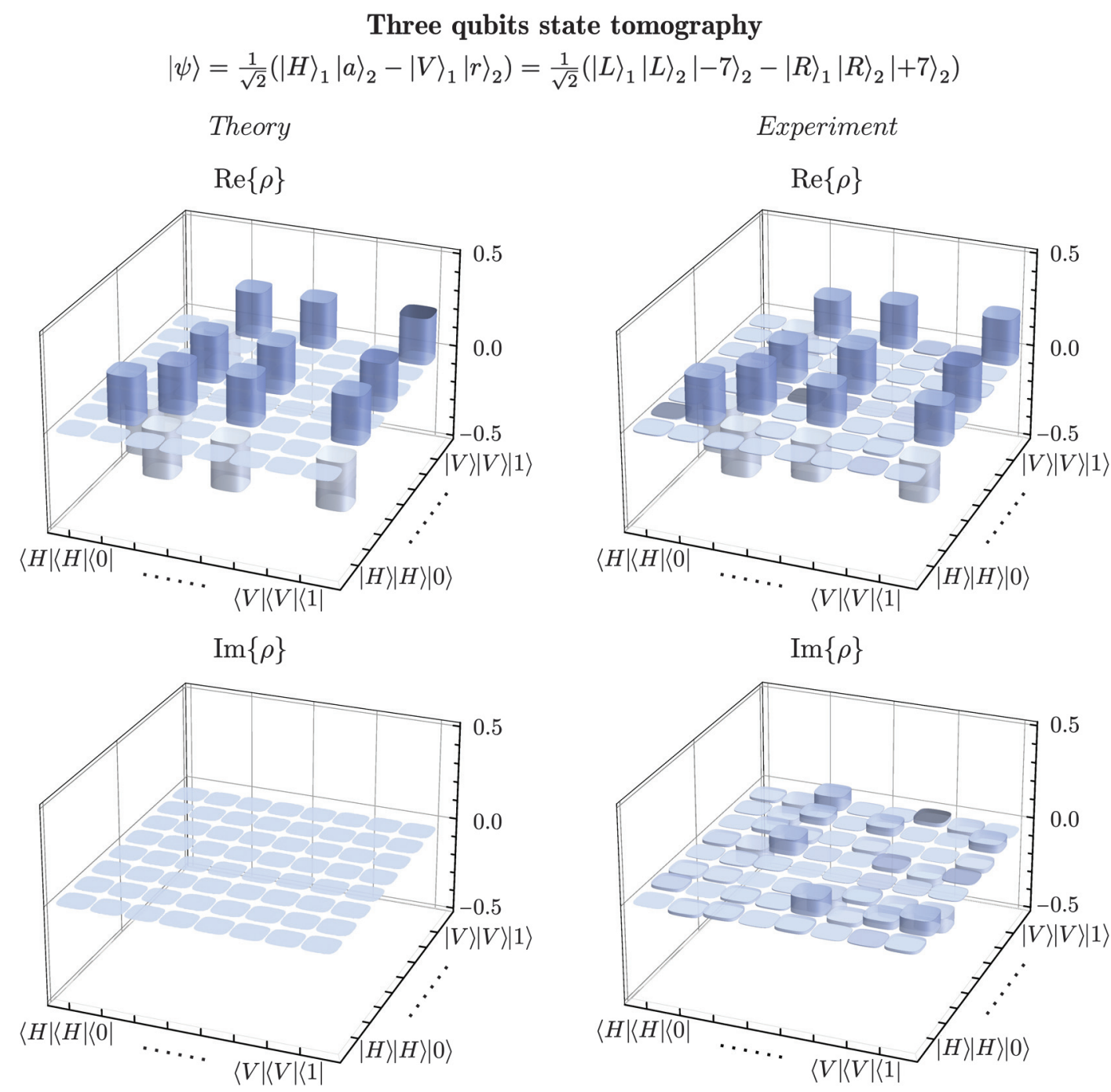

Fig. 5 Three-qubit quantum tomography. Real and imaginary parts of the measured density matrix of the hybrid VV-polarization state in space $\left\{|p o l\rangle_{1}|p o l\rangle_{2}|o a m\rangle_{2}\right\}$ after the fiber transmission (right) and of the theoretical density matrix of state in Eq. (4) (left). The OAM states $|0\rangle$ and $|1\rangle$ in the tomography are defined by the relations: $|0\rangle \equiv(|+7\rangle+|-7\rangle) / \sqrt{2}$ and $|1\rangle \equiv$ $i(|-7\rangle-|+7\rangle) / \sqrt{2}$. Real and imaginary parts of the experimental density matrices are reconstructed via quantum state tomography.

where $A_{i}\left(B_{i}\right)$ represents a dichotomic operator $A(B)$ acting on qubit $i=1,2,3$ with eigenvalues $\pm 1, \bar{B}_{i} \equiv-B_{i}$ and the probabilities $P\left(X_{1} Y_{2} Z_{3}\right) \equiv P\left(X_{1}=1, Y_{2}=1, Z_{3}=1\right)$. In our case, the transmitted three-qubit state permits one to maximally violate the generalized Hardy test by choosing the operators: $A_{1}=$ $A_{2}=-A_{3}=\sigma_{z}$ and $B_{1}=B_{2}=B_{3}=\sigma_{x}$ relative to the qubits $|p o l\rangle_{1}$ and $|p o l\rangle_{2}$ (in basis $\{|H\rangle,|V\rangle\}$ ) and $|o a m\rangle_{2}$ (in basis $\{|0\rangle,|1\rangle\})$, respectively. The experimental value $\mathcal{H}$ obtained for raw data is $\mathcal{H}^{(\text {raw })}=0.085 \pm 0.008$ and by accounting for accidental coincidences it becomes $\mathcal{H}=0.104 \pm 0.008$ (theoretical value for the ideal state is $\mathcal{H}=0.25$ ). Such values allow one to violate the noncontextual bound by 10 and 12 standard deviations, respectively. Note that the tripartite correlations obtained are generated by both contextual (intrasystem) and nonlocal (intersystem) entanglement. Thus, the correlations lie between three qubits and not between three different and spatially separated parties.

\section{Conclusions and Discussion}

Future quantum communication will require the distribution of quantum states over long distances. The protocols implemented within such systems will include the distribution of high-dimensional and entangled quantum states. Indeed, spanning Hilbert spaces of greater dimensions allows higher information capacity and noise resilience, leading to enhanced quantum information processing. ${ }^{38,86,87}$ In this context, VV states represent a powerful resource for classical and quantum applications.

Here, we demonstrated the feasibility of distributing complex VV states through an OAM supporting fiber, also permitting one to preserve entanglement with a different system. In particular, we achieved the transmission of a VV state, presenting correlations between polarization and OAM, entangled with the polarization of a separate second photon. To fully assess the robustness to decoherence and quality of the transmitted complex 
entangled state, we performed quantum state tomographies, violations of CHSH-like inequalities and multipartite entanglement tests. The achieved fidelities of the transmitted state demonstrate the capability to perform high fidelity distribution in an OAM supporting fiber of a hybrid VV-polarization entangled state at telecom wavelength. In particular, the possibility of simultaneously encoding and distributing information in the polarization and OAM degree of freedom of a single particle represents a useful resource due to the higher robustness to losses while tools for their processing have been identified. ${ }^{88}$ This work paves the way toward adoption of high-dimensional entanglement in quantum networks. Further perspectives of this work involve the investigation of fiber-based distribution of different orders of OAM entangled states and their distribution over longer distances, exploiting the potential scalability arising from a fiber-based approach. Indeed, the results presented here are expected to be extended to long distance transmission since low mode mixing can be achieved in longer fiber. ${ }^{47}$ Other perspectives involve interfacing of OAM integrated circuits ${ }^{89-91}$ through OAM supporting fibers for future quantum networks.

Note: During the preparation of this manuscript, the authors became aware of a work by Huan Cao et al. on a similar topic. ${ }^{92}$

\section{Acknowledgments}

We thank S. Ramachandran and P. Gregg for the fiber design, P. Kristensen from OFS-Fitel for the fiber fabrication, and D. Poderini for many advices on the software development. Funding Information: This work was supported by the Center of Excellence, SPOC - Silicon Photonics for Optical Communications (ref DNRF123), by the People Programme (Marie Curie Actions) of the European Union's Seventh Framework Programme (FP7/2007-2013) under REA grant agreement no. 609405 (COFUNDPostdocDTU), and by the ERC-Advanced grant PHOSPhOR (Photonics of Spin-Orbit Optical Phenomena; Grant Agreement No. 694683). G.C. acknowledges Becas Chile and Conicyt.

\section{References}

1. N. Gisin and R. Thew, "Quantum communication," Nat. Photonics 1(3), 165-171 (2007).

2. J.-W. Pan et al., "Experimental entanglement swapping: entangling photons that never interacted," Phys. Rev. Lett. 80(18), 3891-3894 (1998).

3. X.-S. Ma et al., "Experimental delayed-choice entanglement swapping," Nat. Phys. 8(6), 479-484 (2012).

4. M. M. Weston et al., "Heralded quantum steering over a high-loss channel," Sci. Adv. 4(1), e1701230 (2018).

5. Q.-C. Sun et al., "Entanglement swapping with independent sources over an optical-fiber network," Phys. Rev. A 95(3), 032306 (2017).

6. J. T. Barreiro, T.-C. Wei, and P. G. Kwiat, "Beating the channel capacity limit for linear photonic superdense coding," Nat. Phys. 4(4), 282-286 (2008).

7. B. P. Williams, R. J. Sadlier, and T. S. Humble, "Superdense coding over optical fiber links with complete Bell-state measurements," Phys. Rev. Lett. 118(5), 050501 (2017).

8. C. Schuck et al., "Complete deterministic linear optics Bell state analysis," Phys. Rev. Lett. 96(19), 190501 (2006).

9. M. Barbieri et al., "Complete and deterministic discrimination of polarization Bell states assisted by momentum entanglement," Phys. Rev. A 75(4), 042317 (2007).
10. D. Bouwmeester et al., "Experimental quantum teleportation," Nature 390, 575-579 (1997).

11. D. Boschi et al., "Experimental realization of teleporting an unknown pure quantum state via dual classical and EinsteinPodolsky-Rosen channels," Phys. Rev. Lett. 80(6), 1121-1125 (1998).

12. E. Lombardi et al., "Teleportation of a vacuum-one-photon qubit," Phys. Rev. Lett. 88(7), 070402 (2002).

13. X.-L. Wang et al., "Quantum teleportation of multiple degrees of freedom of a single photon," Nature 518(7540), 516-519 (2015).

14. I. Marcikic et al., "Long-distance teleportation of qubits at telecommunication wavelengths," Nature 421(6922), 509-513 (2003).

15. L.-M. Duan et al., "Long-distance quantum communication with atomic ensembles and linear optics," Nature 414(6862), 413-418 (2001).

16. X.-S. Ma et al., "Quantum teleportation over 143 kilometres using active feed-forward," Nature 489(7415), 269-273 (2012).

17. A. Einstein, B. Podolsky, and N. Rosen, "Can quantum-mechanical description of physical reality be considered complete?" Phys. Rev. 47(10), 777-780 (1935).

18. J. S. Bell, "On the Einstein-Podolsky-Rosen paradox," Phys. Phys. Fiz. 1(3), 195 (1964).

19. R. Horodecki et al., "Quantum entanglement," Rev. Mod. Phys. 81(2), 865-942 (2009).

20. M. A. Nielsen and I. L. Chuang, Quantum Computation and Quantum Information, Cambridge University Press, Cambridge (2000).

21. N. Brunner et al., "Bell nonlocality," Rev. Mod. Phys. 86(2), 419478 (2014).

22. G. Adesso, T. R. Bromley, and M. Cianciaruso, "Measures and applications of quantum correlations," J. Phys. A: Math. Theor. 49(47), 473001 (2016).

23. J. Yin et al., "Satellite-based entanglement distribution over 1200 kilometers," Science 356(6343), 1140-1144 (2017).

24. R. Ursin et al., "Entanglement-based quantum communication over 144 km," Nat. Phys. 3(7), 481-486 (2007).

25. T. Honjo et al., "Long-distance entanglement-based quantum key distribution over optical fiber," Opt. Express 16(23), 19118-19126 (2008).

26. T. Inagaki et al., "Entanglement distribution over $300 \mathrm{~km}$ of fiber," Opt. Express 21(20), 23241-23249 (2013).

27. H. Hübel et al., "High-fidelity transmission of polarization encoded qubits from an entangled source over $100 \mathrm{~km}$ of fiber," Opt. Express 15(12), 7853-7862 (2007).

28. A. Poppe et al., "Practical quantum key distribution with polarization entangled photons," Opt. Express 12(16), 3865-3871 (2004).

29. F. Flamini, N. Spagnolo, and F. Sciarrino, "Photonic quantum information processing: a review," Rep. Progr. Phys. 82, 016001 (2019).

30. L. Allen et al., "Orbital angular momentum of light and the transformation of Laguerre-Gaussian laser modes," Phys. Rev. A 45(11), 8185-8189 (1992).

31. G. Molina-Terriza, J. P. Torres, and L. Torner, "Twisted photons," Nat. Phys. 3(5), 305-310 (2007).

32. N. Bozinovic et al., "Terabit-scale orbital angular momentum mode division multiplexing in fibers," Science 340(6140), 1545-1548 (2013).

33. J. Wang et al., "Terabit free-space data transmission employing orbital angular momentum multiplexing," Nat. Photonics 6(7), 488-496 (2012).

34. A. E. Willner et al., "Optical communications using orbital angular momentum beams," Adv. Opt. Photonics 7(1), 66-106 (2015).

35. K. Ingerslev et al., " 12 mode, wdm, mimo-free orbital angular momentum transmission," Opt. Express 26(16), 20225-20232 (2018).

36. M. Malik et al., "Multi-photon entanglement in high dimensions," Nat. Photonics 10(4), 248-252 (2016). 
37. J. Bavaresco et al., "Measurements in two bases are sufficient for certifying high-dimensional entanglement," Nat. Phys. 14(10), 1032-1037 (2018).

38. M. Erhard et al., "Twisted photons: new quantum perspectives in high dimensions," Light: Sci. Appl. 7(3), 17146 (2018).

39. T. Giordani et al., "Experimental engineering of arbitrary qudit states with discrete-time quantum walks," Phys. Rev. Lett. 122(2), 020503 (2019).

40. A. C. Dada et al., "Experimental high-dimensional two-photon entanglement and violations of generalized Bell inequalities," Nat. Phys. 7(9), 677-680 (2011).

41. M. Krenn et al., "Communication with spatially modulated light through turbulent air across Vienna," New J. Phys. 16(11), 113028 (2014).

42. F. Bouchard et al., "Quantum cryptography with twisted photons through an outdoor underwater channel," Opt. Express 26(17), 22563-22573 (2018).

43. Y. Chen et al., "Underwater transmission of high-dimensional twisted photons over 55 meters," arXiv:1902.01392 (2019).

44. C. Brunet et al., "Design, fabrication and validation of an OAM fiber supporting 36 states," Opt. Express 22(21), 26117-26127 (2014).

45. S. Li and J. Wang, "Supermode fiber for orbital angular momentum (OAM) transmission," Opt. Express 23(14), 18736-18745 (2015).

46. B. Ndagano et al., "Fiber propagation of vector modes," Opt. Express 23(13), 17330-17336 (2015).

47. D. Cozzolino et al., "Orbital angular momentum states enabling fiber-based high-dimensional quantum communication," Phys. Rev. Appl. 11(6), 064058 (2019).

48. A. Sit et al., "Quantum cryptography with structured photons through a vortex fiber," Opt. Lett. 43(17), 4108-4111 (2018).

49. P. Gregg, P. Kristensen, and S. Ramachandran, "Conservation of orbital angular momentum in air-core optical fibers," Optica 2(3), 267-270 (2015).

50. M. R. Dennis, K. O'Holleran, and M. J. Padgett, "Singular optics: optical vortices and polarization singularities," Progr. Opt. 53, 293-364 (2009).

51. Q. Zhan, "Cylindrical vector beams: from mathematical concepts to applications," Adv. Opt. Photonics 1(1), 1-57 (2009).

52. F. Cardano et al., "Polarization pattern of vector vortex beams generated by q-plates with different topological charges," Appl. Opt. 51(10), C1-C6 (2012).

53. G. Milione et al., "Higher-order Poincaré sphere, stokes parameters, and the angular momentum of light," Phys. Rev. Lett. 107(5), 053601 (2011).

54. A. F. Abouraddy and K. C. Toussaint Jr., "Three-dimensional polarization control in microscopy," Phys. Rev. Lett. 96(15), 153901 (2006).

55. B. J. Roxworthy and K. C. Toussaint Jr., "Optical trapping with $\pi$-phase cylindrical vector beams," New J. Phys. 12(7), 073012 (2010).

56. H. Moradi et al., "Efficient optical trapping with cylindrical vector beams," Opt. Express 27, 7266 (2019).

57. V. D'Ambrosio et al., "Photonic polarization gears for ultrasensitive angular measurements," Nat. Commun. 4, 2432 (2013).

58. F. K. Fatemi, "Cylindrical vector beams for rapid polarizationdependent measurements in atomic systems," Opt. Express 19(25), 25143-25150 (2011).

59. A. Büse et al., "Symmetry protection of photonic entanglement in the interaction with a single nanoaperture," Phys. Rev. Lett. 121(17), 173901 (2018)

60. V. Parigi et al., "Storage and retrieval of vector beams of light in a multiple-degree-of-freedom quantum memory," Nat. Commun. 6, 7706 (2015).

61. V. D'Ambrosio et al., "Entangled vector vortex beams," Phys. Rev. A 94(3), 030304 (2016).

62. B. Ndagano et al., "Creation and detection of vector vortex modes for classical and quantum communication," J. Lightwave Technol. 36(2), 292-301 (2018).
63. Y. S. Rumala et al., "Tunable supercontinuum light vector vortex beam generator using a q-plate," Opt. Lett. 38(23), 5083-5086 (2013).

64. V. D'Ambrosio et al., "Complete experimental toolbox for alignment-free quantum communication," Nat. Commun. 3, 961 (2012).

65. G. Vallone et al., "Free-space quantum key distribution by rotation-invariant twisted photons," Phys. Rev. Lett. 113(6), 060503 (2014).

66. G. Carvacho et al., "Experimental investigation on the geometry of GHz states," Sci. Rep. 7, 13265 (2017).

67. F. Bouchard et al., "Experimental investigation of high-dimensional quantum key distribution protocols with twisted photons," Quantum 2, 111 (2018).

68. L. Marrucci, C. Manzo, and D. Paparo, "Optical spin-to-orbital angular momentum conversion in inhomogeneous anisotropic media," Phys. Rev. Lett. 96(16), 163905 (2006).

69. L. Marrucci et al., "Spin-to-orbital conversion of the angular momentum of light and its classical and quantum applications," J. Opt. 13(6), 064001 (2011).

70. E. Nagali et al., "Quantum information transfer from spin to orbital angular momentum of photons," Phys. Rev. Lett. 103(1), 013601 (2009).

71. N. Friis et al., "Entanglement certification from theory to experiment," Nat. Rev. Phys. 1, 72-87 (2019).

72. D. F. James et al., "Measurement of qubits," Phys. Rev. A. 64(5), 052312 (2001)

73. J. F. Clauser et al., "Proposed experiment to test local hiddenvariable theories," Phys. Rev. Lett. 23(15), 880-884 (1969).

74. N. D. Mermin, "Extreme quantum entanglement in a superposition of macroscopically distinct states," Phys. Rev. Lett. 65(15), 1838-1840 (1990).

75. M. Ardehali, "Bell inequalities with a magnitude of violation that grows exponentially with the number of particles," Phys. Rev. A 46(9), 5375-5378 (1992).

76. A. Belinski and D. N. Klyshko, "Interference of light and Bell's theorem," Phys. Usp. 36(8), 653-693 (1993).

77. L. Hardy, "Quantum mechanics, local realistic theories, and Lorentz-invariant realistic theories," Phys. Rev. Lett. 68(20), 2981-2984 (1992).

78. L. Hardy, "Nonlocality for two particles without inequalities for almost all entangled states," Phys. Rev. Lett. 71(11), 1665-1668 (1993).

79. S.-H. Jiang et al., "Generalized Hardy's paradox," Phys. Rev. Lett. 120(5), 050403 (2018).

80. S. Kochen and E. P. Specker, "The problem of hidden variables in quantum mechanics," J. Math. Mech. 17, 59-87 (1967).

81. E. Karimi et al., "Spin-orbit hybrid entanglement of photons and quantum contextuality," Phys. Rev. A 82(2), 022115 (2010).

82. A. Aiello et al., "Quantum-like nonseparable structures in optical beams," New J. Phys. 17(4), 043024 (2015).

83. M. McLaren, T. Konrad, and A. Forbes, "Measuring the nonseparability of vector vortex beams," Phys. Rev. A 92(2), 023833 (2015).

84. D. Collins et al., "Bell-type inequalities to detect true n-body nonseparability," Phys. Rev. Lett. 88(17), 170405 (2002).

85. J.-D. Bancal et al., "Device-independent witnesses of genuine multipartite entanglement," Phys. Rev. Lett. 106(25), 250404 (2011).

86. H. Bechmann-Pasquinucci and W. Tittel, "Quantum cryptography using larger alphabets," Phys. Rev. A 61(6), 062308 (2000).

87. N. J. Cerf et al., "Security of quantum key distribution using d-level systems," Phys. Rev. Lett. 88(12), 127902 (2002).

88. C. Vitelli et al., "Joining the quantum state of two photons into one," Nat. Photonics 7(7), 521-526 (2013).

89. X. Cai et al., "Integrated compact optical vortex beam emitters," Science 338(6105), 363-366 (2012).

90. Y. Chen et al., "Mapping twisted light into and out of a photonic chip," Phys. Rev. Lett. 121(23), 233602 (2018). 
91. J. Liu et al., "Direct fiber vector Eigenmode multiplexing transmission seeded by integrated optical vortex emitters," Light: Sci. Appl. 7(3), 17148 (2018).

92. H. Cao et al., "Distribution of high-dimensional orbital angular momentum entanglement at telecom wavelength over $1 \mathrm{~km}$ OAM fiber," arXiv:1811.12195 (2018).

Daniele Cozzolino is a PhD candidate at the SPOC Centre of the Department of Photonics Engineering at the Technical University of Denmark (DTU). He obtained his BSc and MSc degrees in physics at the University of Naples Federico II. His research interests are focused on quantum information and fundamental physics.

Emanuele Polino is a PhD student working under supervision of Fabio Sciarrino at the Quantum Information Lab in the Physics Department at Sapienza Università di Roma. He graduated in 2016 from Sapienza University of Roma. Currently working on photonic technologies for quantum information tasks and quantum foundations studies.

Mauro Valeri is a PhD student working under supervision of Fabio Sciarrino at the Quantum Information Lab in the physics Department at Sapienza Università di Roma. He graduated in 2017 from Sapienza University of Roma. He is currently working on photonic technologies for quantum information tasks, like quantum metrology on photonic devices, and exploiting machine learning techniques.

Gonzalo Carvacho is currently working on quantum optical manipulation, like the orbital angular momentum of light and its applications in quantum information and communication, and quantum foundations such as Bell-like tests with multiphoton generation. He graduated in 2014 from the Universidad de Concepcion (Chile).
Davide Bacco is MSCA PostDoc at Department of Photonics Engineering at the Technical University of Denmark (DTU). He received his degree in engineering telecommunication in 2011 at the University of Padova, Italy. In 2015 he finished in the same university his PhD on science technology and spatial measures (CISAS). His research interests regard quantum communication and silicon photonics for quantum communications.

Nicolò Spagnolo received his $\mathrm{PhD}$ in 2012 in physical science of matter, with a thesis on experimental multiphoton quantum optical states. He is a temporary researcher in the Department of Physics of Sapienza Università di Roma. His research interests have been focused on quantum information and simulation protocols employing different photonic platforms.

Leif K. Oxenløwe is the group leader of the High-Speed Optical Communications Group at DTU Fotonik, Department of Photonics Engineering, at the Technical University of Denmark (DTU), and the centre leader of the Research Centre of Excellence SPOC (Silicon Photonics for Optical Communications) supported by the Danish National Research Foundation. His research interests are focused on silicon photonics for optical processing and high speed optical communication.

Fabio Sciarrino received his $\mathrm{PhD}$ in 2004 with a thesis in experimental quantum optics. He is a full professor and head of the Quantum Information Lab in the Department of Physics of Sapienza Università di Roma. Since 2013, he has been a fellow of the Sapienza School for Advanced Studies. His main field of research is quantum information and quantum optics, with works on quantum teleportation, optimal quantum machines, fundamental tests, quantum communication, and orbital angular momentum. 